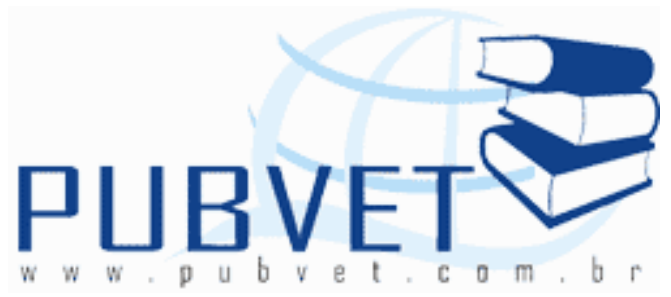

PUBVET, Publicações em Medicina Veterinária e Zootecnia.

\title{
Perfil metabólico, eritrograma, ganho de peso e urinálise de ovelhas superalimentadas e que receberam alimentação balanceada
}

Vanessa Veronese Ortunho ${ }^{1}$, Wilmar Sachetin Marçal ${ }^{2}$, Mara Regina Stipp Balarin $^{2}$

${ }^{1}$ Docente da Universidade Estadual Paulista "Júlio de Mesquita Filho" Campus Ilha Solteira, Departamento de Biologia e Zootecnia e-mail: vanessaverort@yahoo.com.br

2 Docentes da Universidade Estadual de Londrina, Departamento de Clínica Veterinária-CCA

\section{Resumo}

Os objetivos deste trabalho foram verificar se a supernutrição altera o perfil metabólico, o ganho de peso ( $\mathrm{Gpm})$, o eritrograma e a urinálise de borregas. 0 sistema adotado foi o confinamento, a água e o sal mineral foram fornecidos ad libitum. Foram utilizadas 8 fêmeas ovinas da raça Suffolk, as quais foram separadas aleatoriamente em dois grupos, 4 receberam alimentação seguindo os padrões estabelecidos pelo NRC e 4 receberam $110 \%$ do recomendado. Foram realizadas 8 colheitas de sangue, urina e pesagens mensais. Nenhum dos parâmetros analisados sofreram influência da dieta.

Palavras-chave: ovelhas, sangue, urina. 


\title{
Metabolic profile, eritrogram, weight gain and urine analyze by overnutritioned sheep and balanceaded feed
}

\begin{abstract}
The objectives of this study were to determine if the overnutrition alters the metabolic profile, weight gain, the eritrogram and urinalysis. The system adopted was the confinement, the water and mineral salt was supplied ad libitum. The objects of the study were 8 Suffolk ewes randomly distributed in two groups of 4 females which received the standards feed and 4 received $110 \%$ of the recommended. All animals had monthly 8 blood and urine collected for evaluations and none of them were influenced by diet.
\end{abstract}

Keywords: sheep, blood, urine.

\section{INTRODUÇÃO}

A análise do perfil metabólico é extremamente importante para diagnosticar patologias, acompanhar tratamentos, detectar lesões teciduais e transtornos no funcionamento de órgãos, através de análises sanguíneas, no leite, na urina e na saliva (GONZÁLEZ; SCHEFFER, 2003); (RICCÓ, 2004).

Sabe-se que a composição do sangue não é estática e que há grandes variações nos parâmetros em função de fatores como raça, idade, nível de produção, manejo, clima, jejum, estresse e estado fisiológico do animal.

A alimentação que os animais recebem também podem alterar os resultados dos parâmetros sanguíneos. Animais que recebem dieta rica em carboidratos possuem um aumento na concentração de propionato e hiperinsulinemia (ARAI, 2001 apud TANAKA et al., 2008). A redução dos níveis de uréia plasmática pode ocorrer pela redução da proteína dietética e hipoproteinemia (LOPES; BIONDO; SANTOS, 2007). Enquanto que, um nível 
de uréia alto indica excesso de proteína dietética ou um déficit energético (RICCÓ, 2004).

Um manejo alimentar racional e econômico é fundamental para evitar a ocorrência de distúrbios metabólicos, abortos ou diminuição do peso ao nascer dos cordeiros, aumento na taxa de mortalidade, diminuição da produção de leite (PILAR; PEREZ; SANTOS, 2002; SANSON; MOMBACH; SANTOS, 2010; VIÑOLES et al., 2005).

A nutrição adequada também influencia a puberdade nos ovinos e sabe-se que esse é um importante fenômeno reprodutivo e que adiantá-lo é um dos objetivos dos programas de seleção, pois se reduz o intervalo entre as gerações e acelera os progressos genéticos (BATHAEI; LEROY, 1997).

Os objetivos do trabalho foram: avaliar se a nutrição diferenciada altera os valores do perfil metabólico, eritrograma, urinálise e o ganho de peso de borregas Suffolk criadas no interior do Paraná.

\section{Material e Métodos}

O experimento foi realizado numa propriedade rural localizada no

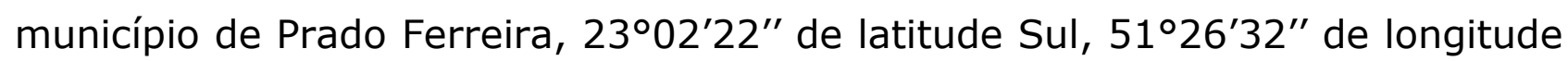
Oeste e $651 \mathrm{~m}$ de altitude, norte do Paraná, Brasil (IPARDES, 2007).

Foram utilizadas 8 fêmeas ovinas da raça Suffolk, as quais foram separadas aleatoriamente em dois grupos, 4 receberam alimentação seguindo os padrões estabelecidos pelo NRC sendo chamadas de grupo $\mathrm{N}$ e 4 receberam $110 \%$ do recomendado chamadas de grupo S.

As fêmeas começaram a receber a dieta aos 4 meses de idade (novembro de 2007), com pesos iniciais de $18,75 \pm 3,77 \mathrm{~kg}$ para as fêmeas do grupo $\mathrm{N}$ e $26 \pm 6,05 \mathrm{~kg}$ para as fêmeas do grupo $\mathrm{S}$. O sistema adotado foi o confinamento e além da ração, era oferecido sal mineral e água a vontade nos cochos. 

peso e urinálise de ovelhas superalimentadas e que receberam alimentação balanceada. PUBVET, Londrina, V. 7, N. 10, Ed. 233, Art. 1538, Maio, 2013.

Foram realizadas 8 colheitas de sangue, urina e pesagens. A primeira colheita teve início após 3 meses da introdução da dieta (fevereiro de 2008), as demais foram realizadas mensalmente, sendo que a última foi realizada em setembro de 2008.

As colheitas de sangue foram realizadas após jejum over-night, através de venopunção da jugular utilizando o sistema a vácuo com agulhas BD Vacutainer $\AA$ descartáveis 22G X 1", adaptador para tubos de colheita. Foram usados 3 tipos de tubos para colheita de sangue: tubo de $5 \mathrm{ml}$ com tampa cinza para análise de glicose sanguínea, tubo de $5 \mathrm{ml}$ com tampa roxa para a realização do eritrograma e tubo de $10 \mathrm{ml}$ com tampa vermelha para que fosse retirado o soro.

O transporte dos tubos de tampa vermelha foi realizado em estantes verticais, sendo que o conjunto estante-tubos foi colocado numa inclinação de aproximadamente $45^{\circ}$ para facilitar a separação do soro, enquanto que os outros tubos foram transportados em caixas de isopor até o laboratório.

As amostras que estavam nos tubos de tampa cinza e roxa foram transportadas até o laboratório em caixas de isopor contendo gelo.

As amostras que estavam nos tubos de tampa cinza foram centrifugadas em alta rotação por 10 minutos para obtenção do plasma. A concentração da glicose plasmática foi obtida nos mesmos dias em que as colheitas foram realizadas por espectrofotometria no aparelho Bioplus, utilizando kit de metodologia enzimática colorimétrica da Gold Analisa.

As amostras que estavam nos tubos de tampa roxa foram analisadas no mesmo dia da colheita e os eritrogramas foram feitos no aparelho $\mathrm{MS}_{4}$.

As amostras contidas nos tubos de tampa vermelha foram centrifugadas por 10 minutos para obtenção do soro, o qual foi colocado em tubos de eppendorf e congelados a $-10 C^{\circ}$ para posteriores análises. A concentração do Aspartato Transaminase (AST), Fosfatase Alcalina (FA),Gama- 
glutamil transferase (GGT) e de creatinina foi obtida por espectrofotometria utilizando metodologia cinética colorimétrica do kit comercial da Gold Analisa.

A concentração do $\beta$ - hidroxibutirato (BHB) foi obtida por espectrofotometria no aparelho Bioplus, utilizando metodologia enzimática cinética kit da marca Randox.

A concentração da albumina plasmática e proteína total (Ptotal) foram obtidas por espectrofotometria utilizando metodologia colorimétrica, a concentração de uréia plasmática, colesterol (col) total, colesterol (col) HDL e triglicérides (trigli) foi realizada utilizando metodologia enzimáticacolorimétrica, todos da marca Gold Analisa

As urinas foram coletadas conforme descrito na literatura e foram armazenadas em potes apropriados e encaminhados ao laboratório em caixas de isopor contendo gelo. As análises foram feitas no mesmo dia das colheitas para que não houvesse alteração no material. Foram realizadas as análises físicas, químicas e de sedimentos.

Todas as amostras foram analisadas no Laboratório de Patologia Clínica do Hospital Veterinário da Universidade Estadual de Londrina.

As pesagens foram feitas nos mesmos dias em que as colheitas de sangue e de urina foram realizadas, para que pudesse ser analisado o ganho de peso médio (Gpm).

Os dados obtidos foram avaliados através do programa SAEG (UFV, 2007).

\section{Resultados e Discussão}

Como pode ser observado pelas Tabelas 1, 2, 3 e 4 nenhum dos parâmetros analisados sofreram influência dos tratamentos $(P>0,05)$.

Todos os valores encontrados durante o experimento concordam com os valores de referência para a espécie segundo Kaneko (1997). 
ORTUNHO, V.V., MARÇAL, W.S. e BALARIN, M.R.S. Perfil metabólico, eritrograma, ganho de peso e urinálise de ovelhas superalimentadas e que receberam alimentação balanceada. PUBVET, Londrina, V. 7, N. 10, Ed. 233, Art. 1538, Maio, 2013.

Tanaka et al. (2008) realizaram experimentos semelhantes, mas utilizaram fêmeas prenhes e encontraram que as fêmeas que estavam subnutridas tiveram menores valores de glicose plasmática, maiores valores de BHB, menores ganhos de peso. Porém também não encontraram diferença nos valores de proteína total, triglicérides, colesterol, AST. O fato destes autores terem encontrado diferença pode ser explicado pelo fato que ovlehas prenhes são mais sensíveis a alterações na dieta.

Tabela 1- Perfil metabólico de borregas que receberam alimentação balanceada e superalimentadas.

\begin{tabular}{|c|c|c|c|c|c|c|c|c|c|}
\hline Colheitas & $1^{a}$ & $2^{a}$ & $3^{a}$ & $4^{a}$ & $5^{a}$ & $6^{a}$ & $7^{a}$ & $8^{a}$ & Médias \\
\hline $\begin{array}{l}\text { Glicose }(\mathrm{mg} / \mathrm{dl}) \\
\mathrm{S} \\
\mathrm{N}\end{array}$ & $\begin{array}{l}50,4 \\
47,12\end{array}$ & $\begin{array}{l}74,15 \\
65,35\end{array}$ & $\begin{array}{l}60 \\
58,07\end{array}$ & $\begin{array}{l}53,75 \\
65,17\end{array}$ & $\begin{array}{l}65,55 \\
64,57\end{array}$ & $\begin{array}{l}56,25 \\
55,75\end{array}$ & $\begin{array}{l}89 \\
86\end{array}$ & $\begin{array}{l}67,05 \\
73,12\end{array}$ & $\begin{array}{l}64,53 \\
64,4\end{array}$ \\
\hline $\begin{array}{l}\text { Albumina }(\mathrm{g} / \mathrm{dl}) \\
\mathrm{S} \\
\mathrm{N}\end{array}$ & $\begin{array}{l}3,35 \\
2,92\end{array}$ & $\begin{array}{l}2,88 \\
2,82\end{array}$ & $\begin{array}{l}2,77 \\
2,77\end{array}$ & $\begin{array}{l}2,3 \\
2,02\end{array}$ & $\begin{array}{l}5,47 \\
4,27\end{array}$ & $\begin{array}{l}2,9 \\
2,65\end{array}$ & $\begin{array}{l}3,47 \\
2,72\end{array}$ & $\begin{array}{l}3,12 \\
2,42\end{array}$ & $\begin{array}{l}3,28 \\
2,82\end{array}$ \\
\hline $\begin{array}{l}\text { AST }(\mathrm{UI} / \mathrm{I}) \\
\mathrm{S} \\
\mathrm{N}\end{array}$ & $\begin{array}{l}153,25 \\
127\end{array}$ & $\begin{array}{l}190,5 \\
184,25\end{array}$ & $\begin{array}{l}359 \\
160,75\end{array}$ & $\begin{array}{l}170 \\
131,75\end{array}$ & $\begin{array}{l}186,25 \\
163,75\end{array}$ & $\begin{array}{l}243,25 \\
190,5\end{array}$ & $\begin{array}{l}227,75 \\
197,25\end{array}$ & $\begin{array}{l}170,25 \\
182,75\end{array}$ & $\begin{array}{l}212,53 \\
167,25\end{array}$ \\
\hline $\begin{array}{l}\mathrm{BHB}(\mathrm{mmol} / \mathrm{l}) \\
\mathrm{S} \\
\mathrm{N}\end{array}$ & $\begin{array}{l}0,21 \\
0,17 \\
\end{array}$ & $\begin{array}{l}0,28 \\
0,23 \\
\end{array}$ & $\begin{array}{l}0,28 \\
0,25 \\
\end{array}$ & $\begin{array}{l}0,18 \\
0,21 \\
\end{array}$ & $\begin{array}{l}0,21 \\
0,19 \\
\end{array}$ & $\begin{array}{l}0,45 \\
0,29 \\
\end{array}$ & $\begin{array}{l}0,45 \\
0,33 \\
\end{array}$ & $\begin{array}{l}0,24 \\
0,18 \\
\end{array}$ & $\begin{array}{l}0,29 \\
0,23 \\
\end{array}$ \\
\hline $\begin{array}{l}\text { Col HDL }(\mathrm{mg} / \mathrm{dl}) \\
\mathrm{S} \\
\mathrm{N}\end{array}$ & $\begin{array}{l}21 \\
20,25\end{array}$ & $\begin{array}{l}12,75 \\
16\end{array}$ & $\begin{array}{l}36 \\
37,25\end{array}$ & $\begin{array}{l}42 \\
39,5 \\
\end{array}$ & $\begin{array}{l}54,75 \\
33,25\end{array}$ & $\begin{array}{l}38,25 \\
45,25\end{array}$ & $\begin{array}{l}33 \\
36\end{array}$ & $\begin{array}{l}44,75 \\
47,5\end{array}$ & $\begin{array}{l}35,31 \\
34,37\end{array}$ \\
\hline $\begin{array}{l}\text { Col total }(\mathrm{mg} / \mathrm{dl}) \\
\mathrm{S} \\
\mathrm{N}\end{array}$ & $\begin{array}{l}43,75 \\
51,25\end{array}$ & $\begin{array}{l}48,25 \\
68,25\end{array}$ & $\begin{array}{l}111,25 \\
101,75\end{array}$ & $\begin{array}{l}95,5 \\
77\end{array}$ & $\begin{array}{l}135,5 \\
133,75\end{array}$ & $\begin{array}{l}82,25 \\
86,75\end{array}$ & $\begin{array}{l}109,75 \\
90,75\end{array}$ & $\begin{array}{l}130,75 \\
97\end{array}$ & $\begin{array}{l}94,62 \\
88,31\end{array}$ \\
\hline $\begin{array}{l}\text { Creatinina } \\
(\mathrm{mg} / \mathrm{dl}) \\
\mathrm{S} \\
\mathrm{N} \\
\end{array}$ & $\begin{array}{l}1,01 \\
1,005\end{array}$ & $\begin{array}{l}0,85 \\
0,95\end{array}$ & $\begin{array}{l}0,97 \\
1,03\end{array}$ & $\begin{array}{l}0,96 \\
0,89 \\
\end{array}$ & $\begin{array}{l}1,06 \\
1,14\end{array}$ & $\begin{array}{l}0,975 \\
1,075 \\
\end{array}$ & $\begin{array}{l}0,825 \\
1,0 \\
\end{array}$ & $\begin{array}{l}0,88 \\
0,97\end{array}$ & $\begin{array}{l}0,94 \\
1,0\end{array}$ \\
\hline $\begin{array}{l}\text { FA }(U \mathrm{UI} / \mathrm{I}) \\
\mathrm{S} \\
\mathrm{N}\end{array}$ & $\begin{array}{l}266 \\
150,75\end{array}$ & $\begin{array}{l}305,75 \\
154,75\end{array}$ & $\begin{array}{l}246,25 \\
181,75\end{array}$ & $\begin{array}{l}216,75 \\
9,25\end{array}$ & $\begin{array}{l}210 \\
154,5\end{array}$ & $\begin{array}{l}240,25 \\
165,75\end{array}$ & $\begin{array}{l}228 \\
198,25\end{array}$ & $\begin{array}{l}210,5 \\
176\end{array}$ & $\begin{array}{l}240,43 \\
159\end{array}$ \\
\hline $\begin{array}{l}\text { GGT (UI/I) } \\
\text { S } \\
\mathrm{N}\end{array}$ & $\begin{array}{l}41,5 \\
31,25\end{array}$ & $\begin{array}{l}40 \\
29,75\end{array}$ & $\begin{array}{l}59 \\
33,25\end{array}$ & $\begin{array}{l}58,5 \\
35\end{array}$ & $\begin{array}{l}41,75 \\
36,75\end{array}$ & $\begin{array}{l}40 \\
28,5\end{array}$ & $\begin{array}{l}38,25 \\
30\end{array}$ & $\begin{array}{l}72,25 \\
30,25\end{array}$ & $\begin{array}{l}48,9 \\
31,84\end{array}$ \\
\hline $\begin{array}{l}\text { Ptotal }(\mathrm{g} / \mathrm{dl}) \\
\mathrm{S} \\
\mathrm{N}\end{array}$ & $\begin{array}{l}6,65 \\
6,65\end{array}$ & $\begin{array}{l}5,2 \\
5,45\end{array}$ & $\begin{array}{l}7,15 \\
7,17\end{array}$ & $\begin{array}{l}5,7 \\
5,65\end{array}$ & $\begin{array}{l}5,47 \\
6,75\end{array}$ & $\begin{array}{l}5,05 \\
4,7\end{array}$ & $\begin{array}{l}6,1 \\
5,9\end{array}$ & $\begin{array}{l}5,6 \\
5,6\end{array}$ & $\begin{array}{l}5,86 \\
5,98\end{array}$ \\
\hline $\begin{array}{l}\text { Trigli }(\mathrm{mg} / \mathrm{dl}) \\
\mathrm{S} \\
\mathrm{N}\end{array}$ & $\begin{array}{l}28,5 \\
31,75\end{array}$ & $\begin{array}{l}28 \\
26,5\end{array}$ & $\begin{array}{l}40,75 \\
38,75\end{array}$ & $\begin{array}{l}47,25 \\
38,5\end{array}$ & $\begin{array}{l}58,75 \\
42,25\end{array}$ & $\begin{array}{l}49,25 \\
48\end{array}$ & $\begin{array}{l}46 \\
36,75\end{array}$ & $\begin{array}{l}47,25 \\
53\end{array}$ & $\begin{array}{l}43,21 \\
39,43\end{array}$ \\
\hline $\begin{array}{l}\text { Uréia }(\mathrm{mg} / \mathrm{dl}) \\
\mathrm{S} \\
\mathrm{N}\end{array}$ & $\begin{array}{l}32 \\
27\end{array}$ & $\begin{array}{l}34,75 \\
30,25\end{array}$ & $\begin{array}{l}48 \\
53,5\end{array}$ & $\begin{array}{l}37,25 \\
35,25\end{array}$ & $\begin{array}{l}55,75 \\
31,25\end{array}$ & $\begin{array}{l}50,5 \\
45,75\end{array}$ & $\begin{array}{l}53 \\
29,25\end{array}$ & $\begin{array}{l}28,5 \\
21\end{array}$ & $\begin{array}{l}42,46 \\
34,15\end{array}$ \\
\hline
\end{tabular}

Fonte: Dados da pesquisa. 
ORTUNHO, V.V., MARÇAL, W.S. e BALARIN, M.R.S. Perfil metabólico, eritrograma, ganho de peso e urinálise de ovelhas superalimentadas e que receberam alimentação balanceada. PUBVET, Londrina, V. 7, N. 10, Ed. 233, Art. 1538, Maio, 2013.

Tabela 2- Eritrograma de borregas que receberam alimentação balanceada e superalimentadas.

\begin{tabular}{l|l|l|l|l|l|l|l|l|l}
\hline Colheitas & $\mathbf{1}^{\mathbf{a}}$ & $\mathbf{2}^{\mathbf{a}}$ & $\mathbf{3}^{\mathbf{a}}$ & $\mathbf{4}^{\mathbf{a}}$ & $\mathbf{5}^{\mathbf{a}}$ & $\mathbf{6}^{\mathbf{a}}$ & $\mathbf{7}^{\mathbf{a}}$ & $\mathbf{8}^{\mathbf{a}}$ & Médias \\
\hline $\begin{array}{l}\text { Hematócrito } \\
\text { S }\end{array}$ & $\begin{array}{l}33,67 \\
\mathrm{~N}\end{array}$ & $\begin{array}{l}31,57 \\
31,37\end{array}$ & $\begin{array}{l}41,2 \\
35,82\end{array}$ & $\begin{array}{l}37,7 \\
29,05\end{array}$ & $\begin{array}{l}36,25 \\
28,5\end{array}$ & $\begin{array}{l}33,5 \\
33\end{array}$ & $\begin{array}{l}30,25 \\
33,5\end{array}$ & $\begin{array}{l}37,37 \\
34,25\end{array}$ & $\begin{array}{l}35,19 \\
32,05\end{array}$ \\
\hline $\begin{array}{l}\text { Hemoglobina } \\
\text { S }\end{array}$ & $\begin{array}{l}11,27 \\
\mathrm{~N}\end{array}$ & $\begin{array}{l}10,57 \\
10,65\end{array}$ & $\begin{array}{l}12,97 \\
17,72\end{array}$ & $\begin{array}{l}11,82 \\
9,07\end{array}$ & $\begin{array}{l}12,08 \\
9,49\end{array}$ & $\begin{array}{l}11,16 \\
10,99\end{array}$ & $\begin{array}{l}10,08 \\
11,16\end{array}$ & $\begin{array}{l}11,16 \\
10,99\end{array}$ & $\begin{array}{l}11,39 \\
11,38\end{array}$ \\
\hline $\begin{array}{l}\text { Eritrócitos } \\
\text { S }\end{array}$ & 9,51 & 9,02 & 11,54 & 10,64 & 8,26 & 7,25 & 6,55 & 8,26 & 8,88 \\
N & 9,33 & 9,19 & 10,42 & 8,7 & 6,17 & 7,14 & 7,25 & 6,17 & 8,04 \\
\hline
\end{tabular}

Fonte: Dados da pesquisa.

Tabela 3- Ganho de peso médio (Gpm) de borregas que receberam alimentação balanceada e superalimentadas.

\begin{tabular}{l|l|l|l|l|l|l|l|l}
\hline Gpm (Kg) & $\begin{array}{l}\text { Fev- } \\
\text { março }\end{array}$ & $\begin{array}{l}\text { Março- } \\
\text { abril }\end{array}$ & $\begin{array}{l}\text { Abril- } \\
\text { maio }\end{array}$ & $\begin{array}{l}\text { Maio- } \\
\text { jun }\end{array}$ & $\begin{array}{l}\text { Jun- } \\
\text { jul }\end{array}$ & $\begin{array}{l}\text { Jul- } \\
\text { ago }\end{array}$ & $\begin{array}{l}\text { Ago } \\
\text {-set }\end{array}$ & Médias \\
\hline $\mathrm{S}$ & 5 & 5,25 & $-3,25$ & 0,5 & 8,5 & 7,75 & 1,75 & 3,64 \\
$\mathrm{~N}$ & 0,5 & 5 & -4 & 0,75 & 3 & 2,5 & 0 & 1,10 \\
\hline
\end{tabular}

Fonte: Dados da pesquisa.

Tabela4- Urinálise das borregas que receberam alimentação balanceada e superalimentadas

\begin{tabular}{l|l|l}
\hline & Superalimentadas & Normais \\
\hline Acetona & Negativo & Negativo \\
Bilirrubina & Negativo & Negativo \\
Densidade & 1020 & 1030 \\
Glicose & Negativo & Negativo \\
Hemácias & Ausentes & Ausentes \\
Hemoglobina & Negativo & Negativo \\
Leucócitos & Raros & Raros \\
pH & 6,3 & 6,7 \\
Proteína & Traços & Traços \\
Urobilinogênio & Negativo & Negativo \\
\hline
\end{tabular}

Fonte: Dados da pesquisa. 
ORTUNHO, V.V., MARÇAL, W.S. e BALARIN, M.R.S. Perfil metabólico, eritrograma, ganho de peso e urinálise de ovelhas superalimentadas e que receberam alimentação balanceada. PUBVET, Londrina, V. 7, N. 10, Ed. 233, Art. 1538, Maio, 2013.

A urinálise mostrou que nenhum animal durante o experimento apresentou déficits nutricionais, patologias e que a dieta não alterou os resultados deste exame, os quais podem ser utilizados por outros autores.

\section{Conclusões}

As ovelhas superalimentadas tiveram o mesmo ganho de peso que as ovelhas que receberam uma dieta seguindo os padrões recomendados pelo NRC.

Os valores do perfil metabólico e do eritrograma não sofreram alteração da dieta.

Os valores encontrados podem ser usados como referência para futuros estudos.

\section{Referências}

BATHAEI, S. S.; LEROY, P. L. Note on age and body weight at puberty in Mehraban Iranian fattailed ewe lambs. Tropical Animal Health Production, Dordrecht, v. 29, p. 55-59, 1997.

GONZÁLEZ, F.H.D., SCHEFFER, J.F.S. (2003) Perfil sanguíneo: ferramenta de análise clínica, metabólica e nutricional. In: González, FH.D., Campos, R. (eds.): Anais do I Simpósio de Patologia Clínica Veterinária da Região Sul do Brasil. Porto Alegre: Gráfica da Universidade Federal do Rio Grande do Sul. p.73-89.

INSTITUTO PARANAENSE DE DESENVOLVIMENTO ECONOMICO E SOCIAL - IPARDES. Área, altitude e coordenadas geográficas, segundo os municípios do Paraná. In: Anuário estatístico do Estado do Paraná. Curitiba, 2007. Disponível em:

<http://www.ipardes.gov.br/anuario_2007/1territorio/tab1_1_1.htm> Acesso em: 20 out. 2008.

KANEKO, J. J. Carbohidrate Metabolism and its disease. In:

domestic animals. 5. ed. New York: Academic Press, 1997. p. 45-81.

Clinical biochemistry of

LOPES, S. T. A; BIONDO, A. W. ; SANTOS, A. P. Manual de Patologia Clínica Veterinária.

3. ed. Santa Maria, 2007. 107 p.

PILAR, R. C.; PEREZ, J.; SANTOS, C. L. Manejo reprodutivo da ovelha recomendações para uma parição a cada 8 meses. Lavras: UFLA, 2002. 32 p. (Boletim Técnico, v. 50).

SANSON, R. M. M.; MOMBACH, M. A.; SANTOS, S. F. Suplementação mineral para fêmeas caprinas e ovinas lactantes. Piracicaba: FarmPoint, 2010. Disponível em:

<http://www.farmpoint.com.br/suplementacao-mineral-para-femeas-caprinas-e-ovinaslactantes_noticia_62422_3_28_.aspx>. Acesso em 1 jun. 2010. 
RICCÓ, D. Indicadores sanguíneos e corporais de avaliação metabólico-nutricional em ruminantes. 2004. 13f. Seminário (Pós-Graduação em Ciências Veterinárias) - Universidade Federal do Rio Grande do Sul, Porto Alegre. Disponível em:

<http://www6.ufrgs.br/bioquimica/posgrad/BTA/perfil_ruminantes.pdf> Acesso em: 30 abr. 2008.

TANAKA, Y. et al. Plasma metabolite concentrations and hepatic enzyme activities in pregnant Romney ewes with restricted feeding. Research in Veterinary Science, Hannover, v. 85, p. 17-21. 2008.

VIÑOLES, C. et al. Short-term nutritional supplementation of ewes in low body condition affects follicle development due to an increase in glucose and metabolic hormones.

Reproduction Research, Uruguay, v. 129, p. 299-309, 2005. 